\title{
ALCANCE: Núcleo de Pesquisas em Acessibilidade, Usabilidade e Linguística Computacional
}

\author{
André Pimenta Freire, Erick Galani Maziero, José Monserrat Neto, Juliana Galvani Greghi, Paula \\ Christina Figueira Cardoso, Rafael Serapilha Durelli, Raphael Winckler de Bettio \\ \{apfreire,erick.maziero,monserrat,juliana,paula.cardoso,rafael.durelli,raphaelwb\}@ufla.br \\ Universidade Federal de Lavras \\ Lavras, MG
}

\begin{abstract}
RESUMO
O Núcleo Alcance é um grupo multidisciplinar que realiza pesquisas em Acessibilidade, Usabilidade e Linguística Computacional, e busca auxiliar as pessoas em tarefas pessoais, educacionais e profissionais com sistemas interativos e processamento de língua natural. $\mathrm{O}$ grupo é membro do Centro Nacional de Referência em Tecnologia Assistiva (CNRTA), do Governo Federal e afiliado à Rede Mineira de Tecnologia Assistiva.
\end{abstract}

\section{PALAVRAS-CHAVE}

Acessibilidade; Usabilidade; Linguística Computacional; Tecnologia Assistiva; Design de Interação

\section{INTRODUÇÃO}

O ALCANCE é um núcleo interdisciplinas de pesquisas fundado no ano de 2013 na Universidade Federal de Lavras. O grupo tem pesquisadores de várias áreas, e atua em pesquisas nas áreas de Acessibilidade, Usabilidade e Linguística Computacional.

O ALCANCE tem como principais linhas de pesquisa:

- Desenvolvimento de Modelos e Recursos de Tecnologia Assistiva

- Métodos para Design e Avaliação de Usabilidade e Acessibilidade de Sistemas Interativos

- Processamento de Língua Natural - PLN

- Utilização de Recursos de Tecnologia Assistiva na Educação

\section{INSTITUIÇÕES PARTICIPANTES}

O grupo é sediado na Universidade Federal de Lavras, com participação de diversos departamentos, como o Departamento de Ciência da Computação, e colaborações com o Departamento de Ciências Exatas, Departamento de Educação, Departamento de Direito, Departamento de Administração e Departamento de Estudos da Linguagem.

Diversos projetos atuais e passados foram desenvolvidos ou estão em desenvolvimento em cooperações com outras instituições, como:

- Associação de Pais e Amigos dos Surdos e Cegos - ASPAC Lavras

Permission to reproduce or distribute, in whole or in part, material extracted from this work, verbatim, adapted or remixed, as well as the creation or production from the content of such work, is granted without fee for non-commercial use, provided that the original work is properly credited.

IHC 2019 - TRILHA FÓRUM DOS GRUPOS DE PESQUISA, Outubro 21-25, 2019 , Vitória, Brasil. In Anais Estendidos do XVIII Simpósio Brasileiro sobre Fatores Humanos em Sistemas Computacionais. Porto Alegre: SBC.

(C) 2019 by the author(s), in accordance with the terms of the Creative Commons Attribution-NonCommercial 4.0 International Public License (CC BY-NC 4.0).
- Associação dos Aposentados, Pensionistas e Idosos de Lavras - AAPIL

- Centro Educacional de Apoio às Necessidades Auditivas e Visuais - CENAV - Lavras

- Instituto Federal de Educação, Ciência e Tecnologia São Paulo em São Carlos

- Queen's University Belfast, UK

- Universidade de São Paulo em São Carlos

- Universidade Federal de Mato Grosso do Sul

- Universidade Federal de Mato Grosso em Rondonópolis

- Universidade Federal de Minas Gerais

- University of York, UK

\subsection{Pesquisadores e Desenvolvedores envolvidos}

Pesquisadores integrantes do Núcleo de Ciência da Computação:

- André Pimenta Freire: Atua em pesquisas na área de acessibilidade em sistemas interativos Web e móveis, com aplicações nas áreas de Educação, Governo e Saúde.

- Erick Galani Maziero: Atua em pesquisas na área de Linguística Computacional, com ênfase análise de discurso e processamento de grandes quantidades de dados textuais.

- José Monserrat Neto: Atua em pesquisas na área de Tecnologia Assistiva, principalmente relacionadas a acesso por pessoas com deficiência visual a conteúdos em imagens.

- Juliana Galvani Greghi: Atua em pesquisas na área de Teste de Software, com ênfase em testes baseados em modelos. Também atua em pesquisas na área de usabilidade e que utilizem o conceito de Internet das Coisas voltadas para Casas Inteligentes Assistivas.

- Paula Christina Figueira Cardoso: Atua em pesquisas na área de Linguística Computacional, com ênfase em algoritmos de sumarização automática de texto. Também atua em pesquisas na área de Informática na Educação.

- Rafael Serapilha Durelli: Tem experiência na área de Ciência da Computação, atuando principalmente nos seguintes temas: Software Product Line, Reuse, Model-Driven Architecture $(M D A)$, Architecture-Driven Modernization (ADM) e Compiler Development.

- Raphael Winckler de Bettio: Possuí experiência na modelagem arquitetural e implementação de sistemas de grande porte. Atua no laboratório como pesquisador no desenvolvimento de tecnologias que utilizem o conceito de Internet das Coisas voltadas para Casas Inteligentes Assistivas. 
Em diferentes projetos, o grupo também tem a colaboração de pesquisadores de outros departamentos.

No projeto "NavMatBR - Recurso para leitura e navegação em fórmulas matemáticas por pessoas com deficiência visual no contexto brasileiro", houve a participação dos professores do Departamento de Ciências Exatas Evelise Roman Corbalan Góis Freire, do setor de Matemática, Rosana Maria Mendes, do setor de Educação Matemática e Helena Libardi, do setor de Ensino de Física. O projeto também conta com a participação da Professora Patricia Vasconcelos Almeida, do Departamento de Estudos da Linguagem. Neste projeto, atualmente atua o aluno de mestrado Hérlon Manollo Cândido Guedes.

Em outros projetos, o grupo também tem colaboração da professora Sílvia Helena Rigatto, do Departamento de Direito. Alunos ligados ao Programa de Pós-Graduação em Administração Pública, na linha de pesquisa de Governo Eletrônico também participam do grupo. Atualmente estão inseridas as alunas de mestrado Luiza Arantes Junqueira e Aline Couto Oliveira. Também há atuação do aluno de mestrado em Ciência da Computação Leonardo Filipe da Silva em projeto ligado a accessibilidade de e-gov para usuários idosos.

No projeto Casa Assistiva, atuam o aluno de mestrado Otávio de Faria Oliveira e os alunos de iniciação científica Lucas Braga Fiorini, Leonardo Henrique de Braz, Luis Felype Fioravanti Ferreira Moreira e Mateus Carvalho Gonçalves.

O laboratório também tem projetos em fase inicial envolvendo o uso de técnicas de Processamento de Língua Natural (PLN) em diferentes aspectos de interação, bem como projetos na área de PLN aplicados a outras áreas. Atualmente, atuam nesta área o aluno de mestrado Jorge Sassaki Resende Silva, e os alunos de iniciação científica Guilherme Henrique Emilioreli Giarola, Guilherme Barbosa Ochikubo, Igor Emanuel Carvalho da Cruz, Ruan Marcos Basílio e Victor Hugo de Andrade Landin.

Em outras áreas de aplicação, o laboratório também tem pesquisas sobre usabilidade em sistemas de saúde. Esses projetos tem o envolvimento do aluno de mestrado Gustavo Lopes Dominguete, e dos alunos de iniciação científica Bruna Santana Capeleti e João Pedro Batista Ferreira.

\section{PRICIPAIS PROJETOS E FOMENTOS RECEBIDOS}

- Usabilidade em sistemas de saúde: O ALCANCE tem estabelecido parcerias com docentes do Departamento de Ciências da Saúde para pesquisa sobre usabilidade em sistemas de saúde, em conjunto com a Profa. Marluce Rodrigues Pereira [3].

- Casa Assistiva: O projeto envolve o design e avaliação [2] de soluções para casas inteligentes com uso de recursos de Internet das Coisas (IoT - Internet of Things), com controle por aplicativos móveis e interações com gestos e comandos de voz, de forma a torná-los acessíveis por pessoas com deficiência e por pessoas idosas. Atualmente, o projeto tem se expandido para incluir questões sobre interação multimodal para pessoas idosas e pessoas com deficiência, com interação por gestos, voz e por aplicativos móveis.
- NavMatBR - Recurso para leitura e navegação em fórmulas matemáticas por pessoas com deficiência visual no contexto brasileiro - Fomento pelo Edital Edital MCTISECIS/CNPq No 20/2016 - Tecnologia Assistiva, em desenvolvimento desde dezembro de 2016. O projeto envolve pesquisa com professores de matemática do Brasil e pessoas com deficiência visual para desenvolver leitura de fórmulas matemáticas na Web em Português do Brasil, com base nas práticas usadas em sala de aula, usando estratégias de Processamento de Língua Natural. Após a finalização do projeto no final de 2018, o produto está em fase de refinamento para disponibilização para a comunidade.

- Mobile Accessibility: Estudo empírico para definição de guidelines de acessibilidade para aplicações móveis baseadas nos problemas encontrados por usuários com deficiência visual - Fomento pelo Edital Universal - MCTI/CNPq No $14 / 2014$, e está em desenvolvimento desde dezembro de 2014. O projeto envolve avaliações de acessibilidade com pessoas com deficiência visual e inspeções técnicas em aplicativos e websites em dispositivos móveis, com o objetivo de indicar recomendações baseadas em problemas encontrados por usuários reais no Brasil.

- Caracterização da inserção dos aspectos de usabilidade e acessibilidade nos processos de desenvolvimento de recursos de Tecnologia Assistiva livres - Fomento pelo Edital FAPEMIG 17/2013 - PPP - Programa Primeiros Projetos, e está em desenvolvimento desde agosto de 2015. O projeto envolve a análise de comunicações entre desenvolvedores e usuários em projetos de software livre de Tecnologia Assistiva, de forma a compilar boas práticas para fomentar o desenvolvimento de TA no Brasil como software livre.

- AudioImagem Sistema Audiodescritor de Imagens Edital - Fomento pelo Edital MCTI-SECIS/CNPq No 84/2013 - Tecnologia Assistiva, desenvolvido entre 2013 e 2015. O objetivo principal do projeto foi o desenvolvimento de um recurso de Tecnologia Assistiva de audiodescrição espacial de imagens, a elaboração de objetos de aprendizagem, com foco em pessoas com deficiência visual [4].

\section{INFRAESTRUTURA}

O ALCANCE dispõe de um laboratório de desenvolvimento e um laboratório de teste de usabilidade. O laboratório de desenvolvimento, com $30 \mathrm{~m}^{2}$, tem 20 postos de trabalho com computadores desktop e laptop, com recursos para apoio a desenvolvimento como mesas digitalizadoras, câmeras de vídeo e de fotografia, rastreador ocular The Eye Tribe, celulares e tablets com sistemas Android e iOS, projetos, dentre outros. Na Figura $1^{1}$, é mostrada uma foto do laboratório e de alguns dos seus membros por ocasião da visita da Rede Mineira de Tecnologia Assistiva, do Governo do estado de Minas Gerais.

O laboratório de teste de usabilidade do ALCANCE tem cerca de $5 \mathrm{~m}^{2}$, e dispõe de duas mesas para testes, com dois computadores desktop, software Morae para análise de testes, webcams e tripé.

${ }^{1}$ Visita da Rede Mineira de Tecnologia Assistiva à UFLA - Disponível em https://goo.gl/9mFcf7 


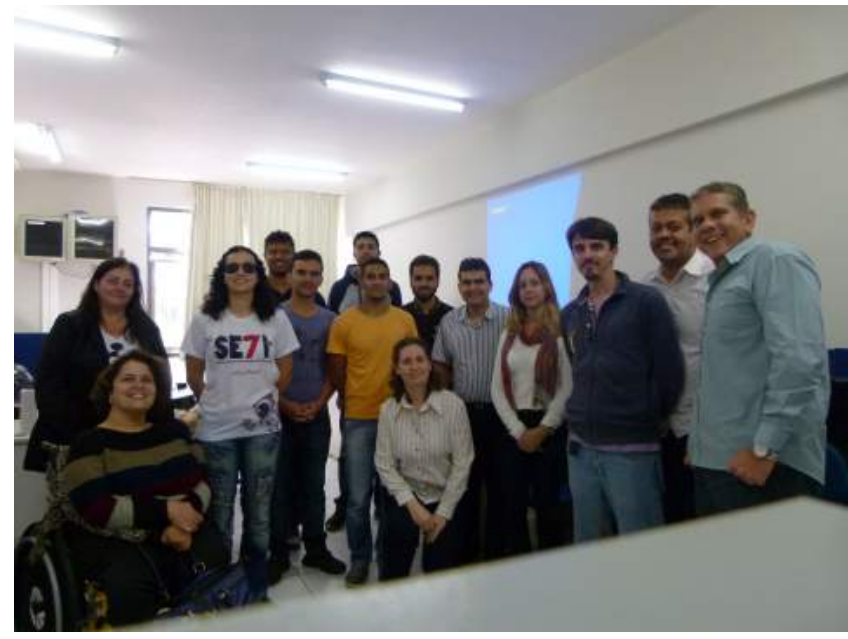

Figura 1: Laboratório de desenvolvimento do ALCANCE em visita da Rede Mineira de Tecnologia Assistiva

A Figura 2 mostra um dos computadores preparado para testes de aplicação com uso de mesa digitalizadora.

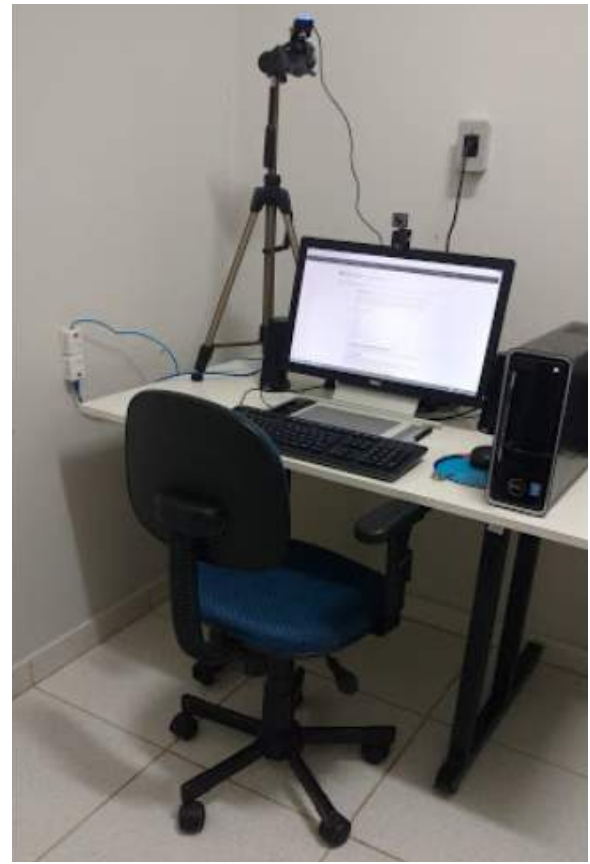

Figura 2: Parte da estrutura da sala do laboratório de teste de usabilidade do ALCANCE

\section{SOLUÇÕES ADOTADAS}

Dos projetos do ALCANCE, o software WebHelpDyslexia [1] é o que teve maior adoção até o momento. $\mathrm{O}$ sistema para apoio à leitura para pessoas com dislexia ou outras dificuldades está disponibilizado livremente na Google Play Store ${ }^{2}$, com mais de 700 downloads e avaliação de 4,8/5. Além disso, o projeto, pós recebimento do prêmio de primeiro lugar na categoria "Aplicativos e Tecnologia Assistiva” do Prêmio Todos@Web, do CGI.br em 2014³ - número de usuários, cobertura na mídia, em jornais de alcance regional e estadual $^{4} 5$.

\section{INTERESSES DE PARCERIA}

O ALCANCE tem interesse em estabelecer parcerias para projetos com empresas e com organizações que trabalham com grupos que representam usuários. O grupo tem possibilidade para parcerias para consultorias e para desenvolvimento de produtos de sistemas interativos. Em particular, o grupo tem grande experiência com o desenvolvimento de recursos de Tecnologia Assistiva para pessoas com deficiência e idosos.

Atualmente, o grupo tem desenvolvido pesquisas relacionadas à usabilidade e acessibilidade de sistemas interativos utilizando tecnologias de Internet das coisas (IoT) e com sensores com aplicações em diversas áreas. Essas pesquisas tem trazido diversos desafios, e temos grande interesse em estabelecer parcerias com outros grupos atuando em pesquisas nessas áreas.

Para realização de projetos em parceria com a indústria, o laboratório tem interesse e disponibilidade para contatos para parcerias. O grupo tem conexão com a Galax, uma organização que apoia a administração e celebração de convênios universidadeempresa para a UFLA. Para maiores informações, consultar o link https://galax.center/ .

\section{ACKNOWLEDGMENTS}

Os autores agradecem pelo financiamento dado pelo CNPq, FAPEMIG, FINEP e FAPEMAT. O grupo tambgradece a todos as pessoas e organizas que colaboram com nossas pesquisas.

\section{REFERÊNCIAS}

[1] Luis Otávio de Avelar, Guilherme Camillo Rezende, and André Pimenta Freire. 2015. WebHelpDyslexia: a browser extension to adapt web content for people with dyslexia. Procedia Computer Science 67 (2015), 150-159.

[2] Gabriela Amaral Araújo de Oliveira, Raphael Winckler de Bettio, and André Pimenta Freire. 2016. Accessibility of the smart home for users with visual disabilities: an evaluation of open source mobile applications for home automation. In Proceedings of the 15th Brazilian Symposium on Human Factors in Computer Systems. ACM, 29.

[3] Camila Rodrigues Dias, Marluce Rodrigues Pereira, and André Pimenta Freire. 2017. Qualitative review of usability problems in health information systems for radiology. Fournal of biomedical informatics 76 (2017), 19-33.

[4] José Monserrat Neto, André Pimenta Freire, Sabrina S Souto, and Ramon S Abílio. 2014. Usability Evaluation of a Web System for Spatially Oriented Audio Descriptions of Images Addressed to Visually Impaired People.. In HCI (5). 154-165.

\footnotetext{
${ }^{2}$ Disponível em https://goo.gl/OyMDAo

${ }^{3} \mathrm{http}: / /$ premio.ceweb.br/2014/

${ }^{4}$ EPTV Sul de Minas: https://goo.gl/7ZPX7P

${ }^{5}$ Bom dia MG: https://goo.gl/Jgy32w
} 